\title{
ATRIBUTOS FÍSICOS DE UM LATOSSOLO VERMELHO DISTROFÉRRICO EM SISTEMA DE INTEGRAÇÃO LAVOURA-PECUÁRIA(1)
}

\author{
Wagner Henrique Moreira( ${ }^{(2)}$, Edner Betioli Junior(2), Leonardo Pim \\ Petean $^{(3)}$, Cássio Antônio Tormena ${ }^{(4)}$, Sérgio José Alves ${ }^{(5)}$, Marco \\ Aurélio Teixeira Costa ${ }^{(2)} \&$ Hélio Henrique Soares Franco(6)
}

\begin{abstract}
RESUMO
O pisoteio dos animais durante o pastejo pode compactar as camadas superficiais e comprometer a qualidade física dos solos em sistema de integração lavoura-pecuária (SILP). A hipótese deste trabalho é de que o pisoteio dos animais decorrente da intensificação do pastejo das culturas de aveia e azevém num Latossolo Vermelho distroférrico com SILP sob plantio direto reduz a qualidade física do solo. Os objetivos deste trabalho foram quantificar a densidade do solo (Ds), a porosidade de aeração $\left(\varepsilon_{\mathrm{a}}\right)$, a permeabilidade do solo ao ar $\left(\mathrm{K}_{a}\right)$ e a resistência do solo à penetração $(\mathrm{RP})$ e estimar índices de continuidade de poros a partir das relações entre $\varepsilon_{\mathrm{a}}$ e a $\mathbf{K}_{a}$. A área estudada, localizada no município de Campo Mourão - PR, foi manejada em SILP com semeadura direta de soja/milho no verão e consórcio de aveia com azevém no inverno, utilizados como forragem para o pastejo pelos animais. Os tratamentos consistiram em quatro alturas de pastejo $(7,14,21$ e $28 \mathrm{~cm})$ e um tratamento controle (testemunha). Em cada tratamento foram coletadas 20 amostras com estrutura indeformada, nas profundidades de 0-7,5 e 7,5-15 cm. Não foram constatadas diferenças significativas na Ds, $\varepsilon_{\mathrm{a}} \mathrm{e}$ $K_{a}$ entre a testemunha e os tratamentos pastejados. Após oito anos de SILP, a Ds, a $\varepsilon_{a}$ e a $K_{\alpha}$ não confirmaram a hipótese de que a intensificação do pisoteio animal reduz a qualidade física do solo. Contudo, a RP mostrou diferenças entre os tratamentos pastejados e a testemunha, com valores restritivos de RP até
\end{abstract}

\footnotetext{
(1) Pesquisa executada com apoio financeiro da Fundação Araucária. Recebido para publicação em 27 de junho de 2011 e aprovado em 19 de janeiro de 2012 .

(2) Programa de Pós-Graduação em Agronomia da Universidade Estadual de Maringá - UEM. Avenida Colombo 5790, CEP 87020900 Maringá (PR). E-mails: wh.moreira@hotmail.com; betioli.jr@gmail.com; marcoatcosta@hotmail.com

(3) Faculdade Integrado de Campo Mourão, Campo Mourão (PR). E-mail: leonardopim@hotmail.com

(4) Professor associado do Departamento de Agronomia, UEM. Bolsista do CNPq. E-mail: catormena@uem.br

(5) Pesquisador do Instituto Agronômico do Paraná (IAPAR). CP 481, CEP 86001-970 Londrina (PR), Brasil. E-mail: sja@iapar.br

(6) Acadêmico de Graduação em Agronomia da Universidade Estadual de Maringá - UEM. E-mail: hhsfranco@hotmail.com
} 
$15 \mathrm{~cm}$ de profundidade, sugerindo que a maior intensidade de pastejo resulta em redução da qualidade física do solo.

Termos de indexação: índices de continuidade de poros, densidade do solo, pisoteio animal, compactação do solo.

\title{
SUMMARY: PHYSICAL PROPERTIES OF AN OXISOL IN AN INTEGRATED CROP-LIVESTOCK SYSTEM
}

\begin{abstract}
The trampling of grazing animals can compact the surface layers and affect the physical quality of soils in crop-livestock systems (ICL). The hypothesis of this study is that increased trampling from animal grazing of oats and ryegrass reduces the soil physical quality of an Oxisol under no-tillage with ICL. The purpose of this study was to quantify bulk density $(B d)$, air-filled porosity ( $(\varepsilon a)$, soil air permeability $(K a)$, soil resistance to penetration $(S R)$ and estimate an index of pore continuity from the linear relationships between $\varepsilon a$ and $K a$. In the experimental area in Campo Mourao - PR, with ICL under no-tillage, soybean or corn was grown as cash crops and oat plus ryegrass as forage for grazing. The treatments were four grazing heights $(7,14,21$, and $28 \mathrm{~cm})$ and an ungrazed control. From each treatment, 20 undisturbed samples were collected from the layers $0-7.5$ and $7.5-15 \mathrm{~cm}$. There were no significant differences in $B d, \varepsilon_{a}$ and Ka between the control and the grazed treatments. In the 0-7.5 cm layer, the mean Ka values were similar between treatments. After eight years of ICL, the BD, $\varepsilon_{a}$ and Ka did not confirm the hypothesis that intensification of grazing reduces the soil physical quality. However, SR showed differences between the control and grazed treatments, with restrictive $S R$ values down to a depth of $15 \mathrm{~cm}$, suggesting that increasing the grazing intensity reduces the soil physical quality.
\end{abstract}

Index terms: index of pore continuity, soil structure, animal trampling, soil compaction.

\section{INTRODUÇÃO}

O sistema de integração lavoura-pecuária (SILP) relaciona diversos fatores biológicos, econômicos e sociais, alternando em um mesmo ano e numa mesma área o cultivo de forrageiras anuais ou perenes a serem utilizadas para a produção de carne e, ou, leite com culturas destinadas à produção de grãos. No sul do Brasil, as espécies usualmente empregadas em SILP são a aveia (Avena strigosa Schreb) e o azevém (Lolium multiflorum Lam.), antecedendo as culturas de soja (Glycine max) ou milho (Zea mays) no verão (Balbinot Junior et al., 2009). O pisoteio dos animais durante o pastejo pode compactar as camadas superficiais do solo e comprometer sua qualidade física, principalmente se realizado com solo úmido e com elevadas taxas de lotação animal (Trein et al., 1991; Bertol et al., 2000; Giarola et al., 2007; Lanzanova et al., 2007). A perda de qualidade física dos solos em SILP pode comprometer a produtividade de grãos e da pastagem, especialmente sob condições climáticas adversas.

Diferentes atributos físicos do solo têm sido utilizados para caracterizar as modificações físicas resultantes da compactação do solo, ocasionadas pela pressão exercida, pelo rodado das máquinas agrícolas, pelo casco dos animais ou, ainda, pelos diferentes sistemas de preparo e manejo do solo. Os mais frequentemente utilizados são a densidade e a porosidade do solo (Albuquerque et al., 2001; Tormena et al., 2002; Flores et al., 2007; Lanzanova et al., 2007), a infiltração de água (Beutler et al., 2001; Lanzanova et al., 2007) e a resistência do solo à penetração (Albuquerque et al., 2001; Tormena et al., 2002; Silva et al., 2003). Em SILP, Lanzanova et al. (2007) constataram que após o pastejo ocorreu aumento de densidade e diminuição da macroporosidade, da porosidade total e da taxa de infiltração de água no solo. Albuquerque et al. (2001) também mostraram aumento da densidade do solo em SILP. Por outro lado, Flores et al. (2007) e Clark et al. (2004) não verificaram alterações na densidade e porosidade do solo em função do pisoteio causado pelos animais. As modificações na estrutura do solo decorrentes da compactação alteram várias características do sistema poroso, afetando o tamanho, a distribuição, a continuidade, o volume e a geometria dos poros (Lima et al., 2005).

A permeabilidade do solo ao ar $\left(K_{a}\right)$ é um atributo físico sensível aos efeitos dos sistemas de uso e manejo na estrutura do solo (Phillips \& Kirkham, 1962) e expressa a habilidade do sistema poroso em relação ao fluxo de gases no solo (Dorner \& Horn, 
2006). Esse atributo tem sido muito utilizado para descrever os efeitos dos sistemas de manejo na estrutura do solo (Ball et al., 1988; Roseberg \& McCoy, 1992) que eventualmente não são capturados pela densidade e, ou, porosidade total do solo. A $\mathrm{K}_{a}$ é dependente da fração do espaço poroso com ar (McQueen \& Shepherd, 2002; Resurreccion et al., 2007) e, portanto, altamente dependente das modificações na distribuição dos macroporos do solo. Dessa forma, alterações em $\mathrm{K}_{a}$ sugerem mudanças na qualidade do ambiente físico para o crescimento de plantas e na taxa de processos dependentes da concentração de gases no solo (Silva et al., 2009). Solos com valores de $\mathrm{K}_{a}=1 \mu \mathrm{m}^{2}$ podem ser considerados impermeáveis, como sugerem McQueen \& Shepherd (2002).

A $\mathrm{K}_{a}$ tem sido utilizada em estudos dos efeitos da compactação sobre a estrutura do solo (Sweeney et al., 2006; Vogeler et al., 2006). As alterações estruturais decorrentes do tráfego de máquinas e de animais, bem como dos sistemas de uso e manejo dos solos e culturas, traduzem-se em modificações na macroporosidade do solo, responsável pelo processo de drenagem e fluxo de gases no solo. Ball (1981), Ball et al. (1988) e McCarthy \& Brown (1992) descrevem uma relação direta entre a $\mathrm{K}_{a}$ e a porosidade de aeração $\left(\varepsilon_{\mathrm{a}}\right)$. Ball et al. (1988), Groenevelt et al. (1984), Blackwell et al. (1990) e Dorner \& Horn (2006) utilizaram as relações entre $K_{a}$ e $\varepsilon_{\mathrm{a}}$ para quantificar modificações funcionais no sistema poroso, como os índices de continuidade de poros. No Brasil, Silva et al. (2009) observaram diminuição da $\mathrm{K}_{a}$ com o aumento da Ds, apresentando valores próximos de $1 \mu \mathrm{m}^{2}$ em Ds próxima a $1.200 \mathrm{~kg} \mathrm{~m}^{-3}$ em solo de classe textural muito argilosa. Já Rodrigues et al. (2011) encontraram valores de aproximadamente $5 \mu \mathrm{m}^{2}$ em solo de classe textural muito argilosa com alto teor de matéria orgânica.

A resistência do solo à penetração (RP) é um dos atributos físicos que mais frequentemente restringem o crescimento das raízes e da parte aérea das plantas, como sugerem Tormena et al. (1999) e Collares et al. (2006). O crescimento radicular é negativamente relacionado com a $R P$, além do fato de esta apresentar forte dependência da Ds e do conteúdo de água do solo $(\theta)$ (Silva et al., 1994; Imhoff et al., 2001). Conte et al. (2007) constataram que os maiores valores de RP decorreram do maior número de animais em pastejo. Para haver melhoria ou manutenção da qualidade física do solo em SILP, deve-se estabelecer uma correta altura de pastejo, via controle da lotação animal, para que não haja compactação do solo devido ao excessivo pisoteio animal (Fidalski et al., 2008). Valores de RP críticos ao desenvolvimento das culturas variam em função da espécie vegetal e das características do solo, como, por exemplo, a composição granulométrica e a estrutura. $\mathrm{O}$ valor de $\mathrm{RP}=2 \mathrm{MPa}$ tem sido amplamente usado por diversos autores como crítico para o crescimento das plantas em diferentes sistemas de manejo (Taylor et al., 1966; Silva et al., 1994; Tormena et al., 1998; Lapen et al., 2004). Petean et al. (2010) utilizaram o valor de 2,5 MPa para solos sob SILP e Tormena et al. (2007) o de $3,5 \mathrm{MPa}$ em solo sob plantio direto, sustentados pelo argumento da presença de bioporos contínuos e efetivos proporcionados por esse sistema de manejo.

A quantificação de medidas que descrevem processos físicos nos solos contribui para o entendimento dos impactos de sistemas de manejo na qualidade física dos solos. Por outro lado, SILPs apresentam complexas interações entre solos, plantas, máquinas e animais, as quais podem ser traduzidas em modificações na estrutura do solo geralmente não completamente capturadas pelos atributos físicos comumente medidos. Os estudos sobre esse tema demonstram uma diversidade de resultados, desde ausência de efeitos até indicações da redução da qualidade física do solo devido ao tráfego de animais em solos sob SILP. Nesse sentido, a hipótese deste trabalho foi de que o pisoteio dos animais em decorrência do pastejo das culturas de aveia e azevém num Latossolo Vermelho distroférrico com SILP sob plantio direto causa alterações que reduzem a qualidade física do solo. Os objetivos deste trabalho foram quantificar a Ds, $\mathrm{RP}, \varepsilon_{\mathrm{a}}$ e $\mathrm{K}_{a}$ e estimar índices de continuidade de poros a partir das relações entre Ka e $\varepsilon_{\mathrm{a}}$ obtidas em amostras indeformadas de solo.

\section{MATERIAL E MÉTODOS}

O experimento foi conduzido no município de Campo Mourão, Paraná, situado a $24^{\circ} 02^{\prime}$ de latitude sul e $52^{\circ} 22^{\prime}$ de longitude oeste. O solo foi um Latossolo Vermelho distroférrico (Embrapa, 2006). A análise granulométrica na camada de $0-20 \mathrm{~cm}$ apresentou valores de 870, 92 e $38 \mathrm{~g} \mathrm{~kg}^{-1}$ de argila, silte e areia, respectivamente. Nessa região, o tipo climático dominante, segundo a classificação de Köppen, é o Cfa (subtropical úmido mesotérmico), caracterizado por temperatura média anual entre 20 e $21^{\circ} \mathrm{C}$ e precipitação pluvial entre $1.600 \mathrm{e}$ $1.800 \mathrm{~mm}$. Os dados de precipitação pluvial do período de 2005 a 2010 são apresentados na figura 1 .

O experimento foi implantado em 2002 em uma área de 8 ha, anteriormente utilizada por 12 anos em sistema de plantio direto. A área em sistema integração lavoura-pecuária (SILP) é cultivada em semeadura direta de soja (Glycine max) ou milho (Zea mays) no verão, com ciclo entre outubro e março, e consórcio de aveia (Avena strigosa Schreb) com azevém (Lolium multiflorum Lam.) no inverno, com ciclo entre maio e outubro, que foram utilizadas como 


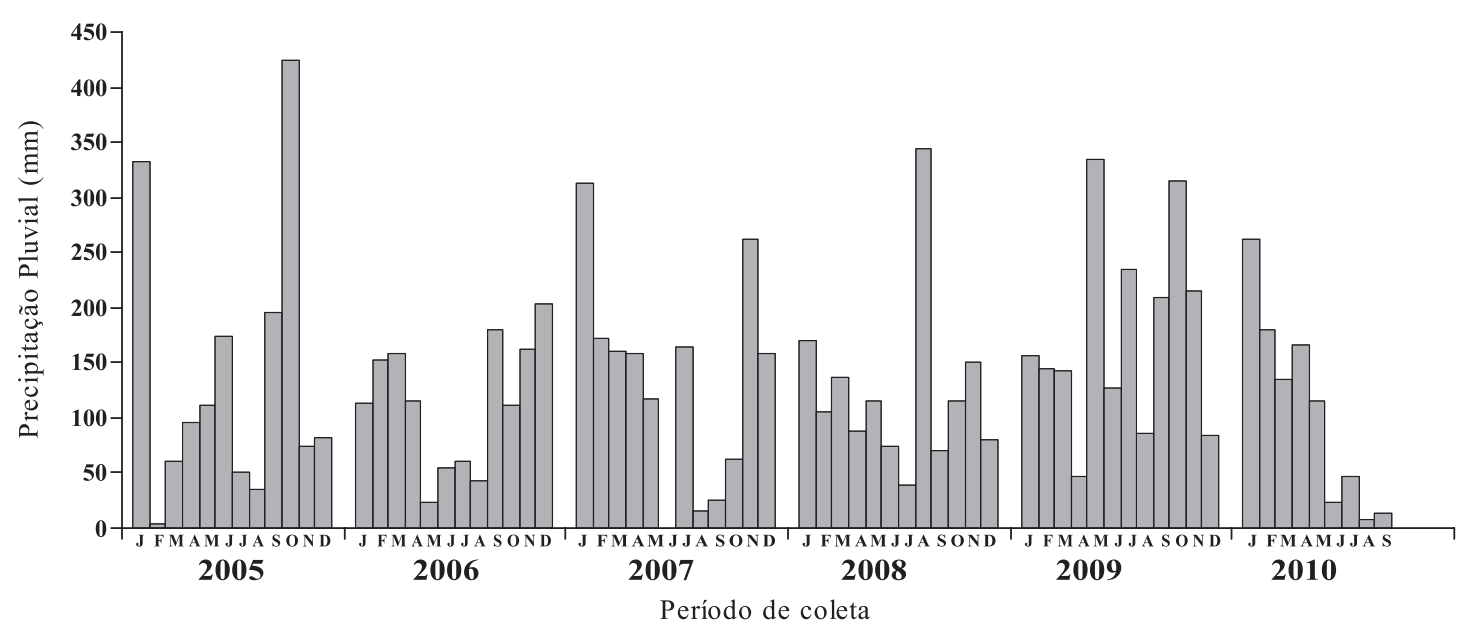

Figura 1. Valores médios mensais de precipitação pluvial na área do experimento. As letras representam as iniciais de cada mês, de janeiro até dezembro.

forragem para o pastejo pelos animais. A semeadura das culturas de verão foi realizada utilizando sulcadores, revolvendo as linhas para deposição de fertilizantes a $10-12 \mathrm{~cm}$ de profundidade, e disco duplo, para deposição das sementes no solo entre 3 e $5 \mathrm{~cm}$. As forrageiras de inverno foram semeadas com semeadora de discos, no espaçamento de $17 \mathrm{~cm}$ entre linhas.

Os tratamentos foram compostos por quatro alturas de manejo das culturas de aveia e azevém: altura de pastejo de $7 \mathrm{~cm}$, com lotação de 2,44 unidades animal (UA) num piquete com área de 1,02 ha; altura de pastejo de $14 \mathrm{~cm}$, com lotação de 2,61 UA num piquete com área de 1,60 ha; altura de pastejo de $21 \mathrm{~cm}$, com lotação de 2,25 UA num piquete com área de 2,16 ha; altura de pastejo de $28 \mathrm{~cm}$, com lotação de 1,82 UA num piquete com área de 3,30 ha; e controle (testemunha) sem pastejo, com área de 0,08 ha. Foi utilizado o método de pastejo contínuo com lotação animal intermitente, put and take (Moot \& Lucas, 1952). Em cada unidade experimental permaneceram dois animais testers e um número variável de animais reguladores, utilizados de forma que se mantivessem as diferentes alturas da pastagem em cada tratamento. Os tratamentos foram delimitados com cerca elétrica, e a altura da pastagem foi monitorada semanalmente, durante $o$ período de cerca de 120 dias.

Em março de 2010, após a colheita da cultura da soja, foram coletadas, aleatoriamente, 20 amostras indeformadas de solo com cilindros metálicos de $100 \mathrm{~cm}^{3}$ (altura e diâmetro de $5 \mathrm{~cm}$ ), em cada tratamento, nas camadas de $0-7,5$ e $7,5-15 \mathrm{~cm}$ de profundidade, pois há maior concentração do sistema radicular entre 0 e $10 \mathrm{~cm}$ (Tardieu 1988). Após a saturação, as amostras foram pesadas e submetidas aos potenciais de -6 e $-10 \mathrm{kPa}$, utilizando uma mesa de tensão similar à descrita por Ball \&
Hunter (1988). Ao atingir o equilíbrio hidráulico, indicado pela ausência de drenagem de água, as amostras foram novamente pesadas; em seguida foi realizada a determinação da $K_{a}$ pelo método da pressão decrescente, utilizando os procedimentos e equipamentos descritos por Silva et al. (2009). As medidas de $\mathrm{K}_{\alpha}$ foram feitas nos potenciais de -6 e $-10 \mathrm{kPa}$, sem ressaturação das amostras após o potencial de $-6 \mathrm{kPa}$. A $\mathrm{K}_{a}\left(\mu \mathrm{m}^{2}\right)$ foi calculada por meio da equação 1 .

$$
K a=(\mu \mathrm{LV} / A P a)|S|
$$

em que $\mu$ é o coeficiente de viscosidade dinâmica do ar $\left(18,1 \times 10^{-6} \mathrm{~kg} \mathrm{~m}^{-1} \mathrm{~s}^{-1}\right.$ a $\left.20^{\circ} \mathrm{C}\right)$; $\mathrm{V}$, o volume do reservatório utilizado $\left(0,01455 \mathrm{~m}^{3}\right)$; $\mathrm{Pa}$, a pressão do ar atmosférico $\left(101.325 \mathrm{~Pa}\right.$ a $\left.20^{\circ} \mathrm{C}\right)$; L, o comprimento da amostra (m); A, a área da amostra $\left(\mathrm{m}^{2}\right)$; e $\mathrm{S}$, o módulo da declividade (ou slope) da equação da reta definida entre as variáveis pressão (P) e Log do tempo (t), cujos dados foram adquiridos pelo sistema automatizado de coleta de dados de pressão e tempo medidos no permeâmetro.

Após a determinação de $\mathrm{K}_{a}$, as amostras foram colocadas em estufa a $\pm 105^{\circ} \mathrm{C}$ por $24 \mathrm{~h}$. A partir da massa do solo seco e do volume de cada cilindro, a densidade do solo, Ds $\left(\mathrm{kg} \mathrm{m}^{-3}\right)$, foi calculada por meio da equação 2, conforme Grossman \& Reinsch (2002).

$$
D s=M s / V t
$$

em que Ms é a massa de sólidos (kg) e Vt, o volume total da amostra $\left(\mathrm{m}^{3}\right)$.

A porosidade de aeração $\left(\varepsilon_{\mathrm{a}}\right)$ foi obtida pela diferença entre a porosidade total (PT) e o conteúdo de água no solo após drenagem das amostras nos potencias de -6 e $-10 \mathrm{kPa}$. A porosidade total foi obtida pela relação entre Ds e Dp (Equação 3). 


$$
P t=1-D s / D p
$$

em que Ds é a densidade do solo $\left(\mathrm{kg} \mathrm{m}^{-3}\right)$ e Dp, a densidade de partículas $\left(\mathrm{kg} \mathrm{m}^{-3}\right)$.

$\mathrm{O}$ conteúdo de água no solo $\left(\theta \mathrm{m}^{3} \mathrm{~m}^{-3}\right)$ foi determinado por meio da equação 4 (Topp \& Ferré, 2002):

$$
\theta=u \times D s
$$

sendo $u$ o teor de água em base de massa $\left(\mathrm{kg} \mathrm{kg}^{-1}\right)$ e Ds, a densidade do solo $\left(\mathrm{kg} \mathrm{m}^{-3}\right)$.

A umidade gravimétrica, $\mathrm{u}\left(\mathrm{kg} \mathrm{kg}^{-1}\right)$, foi obtida por meio da equação 5 :

$$
u=\frac{M u-M s}{M s}
$$

em que $\mathrm{Mu}$ é a massa do solo úmido em cada potencial específico $(\mathrm{kg})$ e Ms, a massa de sólidos do solo $(\mathrm{kg})$.

A relação entre $K_{a}$ e $\varepsilon_{a}$ pode ser utilizada para fazer estimativas de indicadores da continuidade dos poros do solo. Ball et al. (1988) sugerem que $\mathrm{K}_{a}$ e $\varepsilon_{\mathrm{a}}$ podem ser relacionados por um modelo exponencial originalmente proposto por Ahuja et al. (1984) para estabelecer as relações entre as duas variáveis. Em conformidade com Ball et al. (1988), utilizou-se a forma linearizada do modelo exponencial descrita na equação 6 :

$$
\log (K a)=\log (M)+N \log \left(\varepsilon_{\mathrm{a}}\right)
$$

em que $\mathrm{K}_{a}$ é a permeabilidade do solo ao ar $\left(\mu \mathrm{m}^{2}\right) ; \varepsilon_{\mathrm{a}}$, a porosidade de aeração $\left(\mathrm{m}^{3} \mathrm{~m}^{-3}\right)$; e $\mathrm{M}$ (intercepto) e N (inclinação), parâmetros empíricos obtidos no ajuste da equação 6 aos dados. $\mathrm{O}$ valor de $\mathrm{N}$ pode ser tomado como um índice de continuidade de poros, uma vez que $\mathrm{N}$ reflete o aumento de $\mathrm{K}_{a}$ com incremento de $\varepsilon_{\mathrm{a}}$ ou um equivalente decréscimo na tortuosidade dos poros com o acréscimo no volume de poros disponíveis para o fluxo de ar.

As medidas de resistência do solo à penetração (RP) foram realizadas com um penetrômetro eletrônico modelo penetroLOG-Falker- PLG1020. Em cada tratamento foram feitas 20 medidas de $\mathrm{RP}$, aleatoriamente, até a profundidade de $40 \mathrm{~cm}$. Simultaneamente, foram retiradas amostras deformadas de solo a cada $10 \mathrm{~cm}$ de profundidade, para determinação do conteúdo de água gravimétrico do solo.

Os ajustes de equações lineares entre o Log Ka e $\log \varepsilon_{\mathrm{a}}$ foram considerados significativos para $\mathrm{p}<0,05$, e os coeficientes $\mathrm{M}$ e $\mathrm{N}$ foram testados estatisticamente por meio de teste t a $5 \%$. As variáveis $\mathrm{Ds}_{\mathrm{s}} \mathrm{K}_{a}$, $\varepsilon_{\mathrm{a}}$ e $\mathrm{RP}$ foram comparadas entre os tratamentos por meio do uso do intervalo de confiança da média a $95 \%(\mathrm{p}<0,05)$, conforme Payton et al. (2000), considera-se que são estatisticamente diferentes quando não há sobreposição dos limites superior e inferior dos intervalos de confiança das médias comparadas. Na correlação entre as variáveis medidas foi utilizado o teste de correlação de Pearson, avaliando-se o coeficiente de correlação e a probabilidade deste. Todos esses procedimentos foram feitos com o software SAS (SAS, 2002).

\section{RESULTADOS E DISCUSSÃO}

Na camada de 0-7,5 cm, o maior valor de Ds foi constatado no tratamento com altura de pastejo de $28 \mathrm{~cm}$, quando comparado com os tratamentos de altura de pastejo de 7, 14 e $21 \mathrm{~cm}$. Esse resultado pode ter ocorrido em função da maior supressão do azevém pela aveia, já que o desenvolvimento inicial da aveia é mais rápido; devido à altura de pastejo, houve maior sombreamento do azevém nesse tratamento. Não foram verificadas diferenças entre os valores médios de Ds do tratamento sem pastejo (testemunha) em relação aos tratamentos com diferentes intensidades de pastejo, o que é denotado pela sobreposição dos limites do intervalo de confiança da média (Figura 2). Na camada de 7,5-15 cm não foram constatadas diferenças significativas entre os tratamentos (Figura 2). Nesse mesmo experimento, Petean et al. (2009) fizeram medidas de densidade aos três anos após a implantação do experimento e constataram que, na camada de solo de $0-7,5 \mathrm{~cm}$, não haviam diferenças de Ds entre o tratamento testemunha e o tratamento pastejado a $28 \mathrm{~cm}$, corroborando os resultados verificados neste trabalho; por outro lado, verificaram que a Ds no tratamento sem pastejo era inferior àquela dos tratamentos pastejados a 7, 14 e $21 \mathrm{~cm}$ de altura.

Os resultados na figura 2, obtidos oito anos após o início do SILP, indicam que a Ds é similar entre a testemunha e os tratamentos com diferentes alturas de pastejo, tendo como referência os resultados de Petean et al. (2009), em que a densidade da camada de $0-7,5 \mathrm{~cm}$ foi superior estatisticamente à da testemunha nos tratamentos de 7,14 e $21 \mathrm{~cm}$, e sugerem um processo de resiliência da estrutura do solo nos tratamentos com pastejo. Conforme Gregory et al. (2007), a resiliência física do solo é composta por uma série de processos regenerativos, que incluem ciclos de secagem e umedecimento, bem como pelo crescimento de raízes e atividade da mesofauna do solo. O crescimento radicular das gramíneas em consórcio, notadamente do azevém, tanto durante o pastejo como no período pós-pastejo, que antecede a semeadura das culturas de verão, pode ampliar o efeito dos fatores citados por Gregory et al. (2007), favorecendo a regeneração estrutural do solo. 


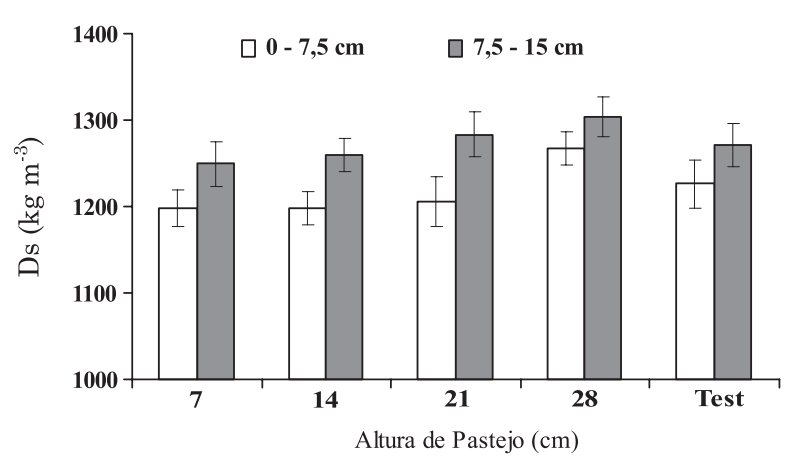

Figura 2. Densidade do solo (Ds) nas camadas de 0-7,5 e 7,5-15 cm para as alturas de pastejo de $7,14,21$ e $28 \mathrm{~cm}$ e testemunha (Test). As barras referem-se ao intervalo de confiança da média, e a sobreposição dos intervalos de confiança indica ausência de diferenças entre as médias dos tratamentos.

A ampliação da resiliência do solo na camada superficial dos tratamentos de 7, 14 e $21 \mathrm{~cm}$ de altura de pastejo pode ser esclarecida comparando os valores de Ds obtidos por Petean et al. (2009), que foram de 1,32, 1,30, 1,27, 1,26 e 1,22 $\mathrm{Mg} \mathrm{dm}^{-3}$, para os tratamentos de 7, 14, 21 e $28 \mathrm{~cm}$ e testemunha, respectivamente, e os mostrados neste trabalho, num intervalo de cinco anos entre as amostragens. Verifica-se que a Ds foi minimamente alterada nos tratamentos testemunha e pastejado a $28 \mathrm{~cm}$ de altura. Esses resultados indicam que o pisoteio animal, mais intenso nos tratamentos de 7, 14 e $21 \mathrm{~cm}$, inicialmente promoveu aumento da Ds e, ao longo do tempo, esta diminuiu.

Entre as camadas estudadas, nos tratamentos e 7,14 e $21 \mathrm{~cm}$ houve diferença estatística da Ds, com a camada de $0-7,5 \mathrm{~cm}$ apresentando menores valores (Figura 2), o que se deve à maior intensificação da secagem e umedecimento do solo, ao revolvimento do solo pelos mecanismos de abertura de sulcos durante a semeadura das culturas de verão e à maior concentração das raízes do azevém nessa camada. A utilização de semeadoras dotadas de sulcadores para aplicação dos fertilizantes quando da semeadura das culturas de soja e milho pode auxiliar na redução da Ds na camada superficial do solo. A efetividade dos sulcadores tem sido indicada por Veiga et al. (2007), sugerindo que eles penetram no solo até cerca de 10-12 cm, o que contribui para aliviar a compactação superficial do solo em sistemas de plantio direto.

Os resultados dos valores médios e do intervalo de confiança da $\varepsilon_{\mathrm{a}}$ obtidos nos potenciais de -6 e $-10 \mathrm{kPa}$ indicam que não houve diferenças estatísticas entre os tratamentos na camada de 0-7,5 cm (Figura 3). Na camada de 7,5-15 cm, por outro lado, verificou-se que a $\varepsilon_{\mathrm{a}}$ nos tratamentos de 7 e $14 \mathrm{~cm}$ foi superior à do tratamento de $28 \mathrm{~cm}$, independentemente do potencial em que ela foi obtida (Figura 3). Essas variações estão associadas com as variações de Ds observadas na figura 2. Quando comparados os valores de $\varepsilon_{\mathrm{a}}$ obtidos no potencial de $-6 \mathrm{kPa}$, que equivalem à macroporosidade do solo, com os obtidos por Petean et al. (2009), que apresentaram aproximadamente $0,06,0,05,0,05,0,08$ e $0,09 \mathrm{~m}^{3} \mathrm{~m}^{-3}$ para os tratamentos de $7,14,21$ de $28 \mathrm{~cm} \mathrm{e}$ testemunha, respectivamente, verifica-se aumento na quantidade de macroporos na camada de $0-7,5 \mathrm{~cm}$ nos tratamentos pastejados com 7, 14 e $21 \mathrm{~cm}$ de altura de pastejo. Já na camada de 7,5-15 cm houve alteração apenas na altura de pastejo de $14 \mathrm{~cm}$, que aumentou de 0,06 para $0,08 \mathrm{~m}^{3} \mathrm{~m}^{-3}$.

Os valores médios de $\varepsilon_{\mathrm{a}}$ na camada de $0-7,5 \mathrm{~cm}$ foram menores do que $0,1 \mathrm{~m}^{3} \mathrm{~m}^{-3}(10 \%)$, considerado o valor mínimo para uma adequada difusão de gases na maioria dos solos, conforme indicações de Grable \& Siemer (1968) e Xu et al. (1992). No entanto, verificou-se que na camada de $0-7,5 \mathrm{~cm}$, no potencial de $-6 \mathrm{kPa}$, a $\varepsilon_{\mathrm{a}}$ variou entre 2,8 e $14,7 \%$, com apenas $12 \%$ das amostras com $\varepsilon_{\mathrm{a}}>10 \%$. Já na camada de $7,5-15 \mathrm{~cm}$, a $\varepsilon_{\mathrm{a}}$ foi maior do que na de $0-7,5 \mathrm{~cm}$, variando de 2,8 a $19,2 \%$ e apresentando $14 \%$ das amostras $\operatorname{com} \varepsilon_{\mathrm{a}}>10 \%$. Assumindo que a condição adequada para a aeração do solo ocorre com $\varepsilon_{\mathrm{a}}$ acima de $10 \%$, os resultados deste estudo indicam que nos potenciais de $-6 \mathrm{kPa}$ a $\varepsilon_{\mathrm{a}}$ não é suficiente, mas aproxima-se desse valor no potencial de $-10 \mathrm{kPa}$
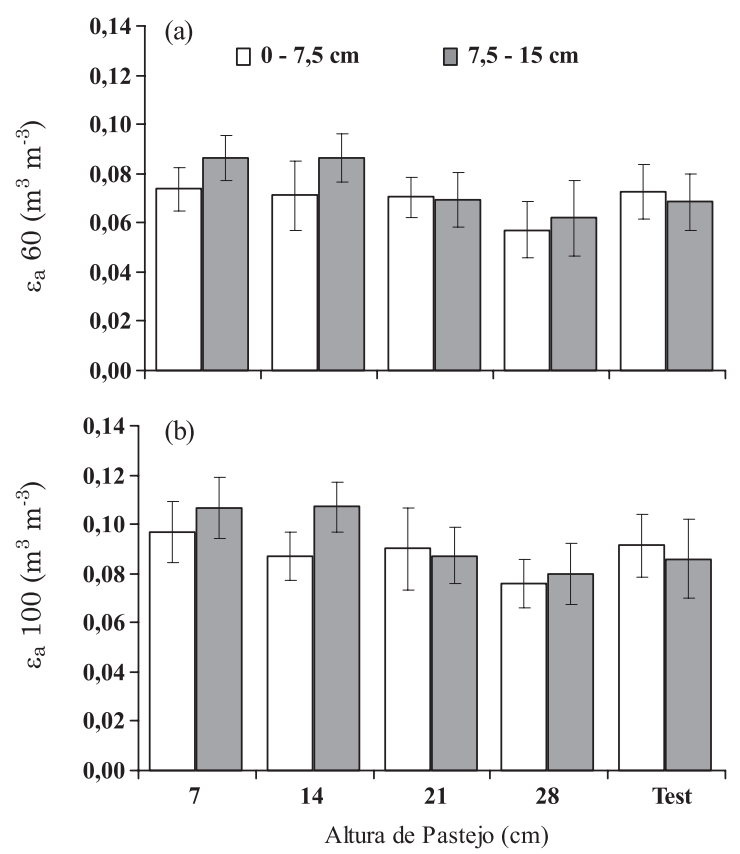

Figura 3. Porosidade com ar no potencial -6 kPa ( $\left.\varepsilon_{\mathrm{a}} 60\right)$, nas camadas de $0-7,5$ e $7,5-15 \mathrm{~cm}$ (a), e no potencial $-10 \mathrm{kPa}\left(\varepsilon_{\mathrm{a}} 100\right)$, nas camadas de 0-7,5 e 7,5-15 cm (b). As barras referem-se ao intervalo de confiança da média, e a sobreposição dos intervalos de confiança indica ausência de diferenças entre as médias dos tratamentos. 
(Figura 3), independentemente dos tratamentos. Esses resultados estão associados ao elevado teor de argila deste solo (870 $\mathrm{g} \mathrm{kg}^{-1}$ ), exigindo maior redução do teor de água ou potencial da água no solo para a drenagem de poros em quantidade suficiente para uma adequada aeração do solo, tomando como base as indicações da literatura. Esses resultados também indicam que, apesar da redução da Ds na camada de 0-7,5 cm dos tratamentos de 7, 14 e $21 \mathrm{~cm}$ em relação ao de $28 \mathrm{~cm}$, não houve aumento significativo da quantidade de poros drenados nos potenciais de -6 e $-10 \mathrm{kPa}$, o qual poderia ser verificado para potenciais menores que $-10 \mathrm{kPa}$.

No potencial de $-10 \mathrm{kPa}$, a maioria dos tratamentos apresentou $\varepsilon_{\mathrm{a}}$ próximo a $10 \%$. A maior Ds na camada de $0-7,5 \mathrm{~cm}$ do tratamento $28 \mathrm{~cm}$ resultou em menores valores médios de $\varepsilon_{\mathrm{a}}$, notadamente abaixo de $10 \%$, indicando que, nesse tratamento, a compactação causada pelos animais não foi aliviada pelos ciclos de secagem e umedecimento, provavelmente menos intensos do que nos outros tratamentos, bem como pela reduzida ação do sistema radicular do azevém, devido à sua menor população nesse tratamento. Os valores de $\varepsilon_{\mathrm{a}}$ obtidos no potencial de $-10 \mathrm{kPa}$ (potencial estimado para a capacidade de campo desse solo) estão aproximadamente no limite para todos os níveis de manejo, sugerindo que há poucos riscos de ocorrer limitação ao desenvolvimento de plantas devido à reduzida aeração do solo, exceto em períodos úmidos prolongados. Contudo, $\varepsilon_{\mathrm{a}}=10 \%$ pode não refletir em medidas precisas do comportamento dos gases no solo, visto que a aeração do solo é um processo dinâmico codependente de outras características do solo e do ambiente, conforme indicam Silva et al. (2009). A hipótese da menor intensidade dos ciclos de secagem e umedecimento e menor atuação das raízes foi estabelecida em razão da reduzida presença de plantas de azevém no tratamento de $28 \mathrm{~cm}$ e do fato de que as plantas de aveia encerram o ciclo de crescimento antes das plantas de azevém. Esses efeitos são similares nos tratamentos de $28 \mathrm{~cm}$ e testemunha, devido ao predomínio de plantas de aveia. A expectativa era de que os tratamentos com maior intensificação de pastejo apresentassem condições físicas mais restritivas; no entanto, a intensificação de renovação de plantas e raízes nesses tratamentos determinou maior recuperação estrutural do solo nesses sistemas de manejo, reduzindo os efeitos danosos da compactação pelos animais, principalmente na camada de $0-7,5 \mathrm{~cm}$.

Os valores médios de $\mathrm{K}_{a}$ foram de $0,143,0,273$, $0,100,0,164$ e $0,142 \mu \mathrm{m}^{2}$ para testemunha, $7,14,21$ e $28 \mathrm{~cm}$ na camada de $0-7,5 \mathrm{~cm}$, respectivamente, e de $0,258,0,362,0,488,0,365,0,687 \mu^{2}$ para testemunha, 7, 14, 21 e $28 \mathrm{~cm}$ na profundidade de 7,5$15 \mathrm{~cm}$, respectivamente. A redução de $\mathrm{K}_{a}$ na camada de $0-7,5 \mathrm{~cm}$ decorre do efeito do pisoteio animal, bem como do tráfego de máquinas, que alteram negativamente a estrutura e as características do sistema poroso do solo, responsáveis pela condução de gases no solo, os quais não foram detectados pela Ds e $\varepsilon_{\mathrm{a}}$. A influência da condição estrutural do solo na $\mathrm{K}_{a}$ foi demonstrada por Ball (1981), que observou redução de duas a três vezes na $\mathrm{K}_{a}$ com a perda da continuidade dos poros. Isso também é reforçado por McCarthy \& Brown (1992), indicando que em solos estruturados a maior $\mathrm{K}_{a}$ é atribuída ao maior espaço disponível para o fluxo de gases em canais interagregados formados entre as unidades estruturais.

Nas camadas de $0-7,5$ e $7,5-15 \mathrm{~cm}$, os valores de $\mathrm{K}_{a}$ não apresentaram diferença estatística entre os tratamentos. Por outro lado, a redução da Ds verificada nesses tratamentos não refletiu em mudança no sistema poroso, especialmente na continuidade de poros, que possibilitasse aumento em $\varepsilon_{\mathrm{a}}$ e $\mathrm{K}_{a}$. A $\mathrm{K}_{a}$ é sensível aos efeitos dos sistemas de uso e manejo na estrutura do solo e pode fornecer uma descrição significativa do estado de compactação do solo (Phillips \& Kirkham, 1962). Como descrito na literatura (Ball, 1981; Resurreccion et al., 2007), a variabilidade da $\mathrm{K}_{a}$ é naturalmente alta. Somado a isso, em experimento de SILP, a heterogeneidade espacial do solo em consequência do pisoteio errático pode aumentar ainda mais a variabilidade da $\mathrm{K}_{a \text {, }}$ o que pode ser a causa da ausência de diferenças estatísticas para a $\mathrm{K}_{a}$ no presente estudo.

$\mathrm{Um}$ valor crítico de $\mathrm{K}_{a}=1 \mu \mathrm{m}^{2}$ tem sido empiricamente estabelecido por Ball et al. (1988) e McQueen \& Shepherd (2002) como indicador de solo impermeável aos gases, sugerindo a ocorrência de condições físicas do solo limitantes para o crescimento das plantas. Verificou-se que $94 \%$ das amostras apresentaram, para o potencial de $-10 \mathrm{kPa}$, valores menores do que esse limite proposto. Comparativamente com outros resultados obtidos na literatura, a exemplo de Ball \& Robertson (1994), o solo em questão é muito argiloso e apresentou reduzida $\varepsilon_{\mathrm{a}}$ no potencial a que as amostras foram submetidas. Resultados similares foram reportados por Silva et al. (2009) em solos de classe textural similar, com valores de $\mathrm{K}_{a}$ próximos de $1 \mu \mathrm{m}^{2}$ para Ds próxima de $1.200 \mathrm{~kg} \mathrm{~m}^{-3}$. Os valores de $\mathrm{K}_{a}$ obtidos por Rodrigues et al. (2011) são muito superiores aos observados neste estudo, atingindo valor médio $\log _{10} \mathrm{~K}_{\alpha}=1,653$ em avaliação da camada superficial de um SPD e em potencial de $-10 \mathrm{kPa}$, tendo em vista os elevados valores de $\varepsilon_{\mathrm{a}}$ obtidos naquele estudo (até $0,38 \mathrm{~m}^{3} \mathrm{~m}^{-3}$ ). Ainda que o solo estudado por Rodrigues et al. (2011) e o deste estudo pertençam à mesma classe textural, o teor de matéria orgânica é muito menor, justificando as diferenças estruturais que possam ter promovido maiores valores de $\mathrm{K}_{a}$, como verificado por Rodrigues et al. (2011). A ocorrência de limitada aeração do solo às plantas está na dependência do tempo em que o solo permanece em potenciais iguais ou maiores 
que $-10 \mathrm{kPa}$ e dos fatores dinâmicos que alteram o balanço de água no solo, sobretudo nas camadas mais superficiais.

Correlações lineares estatisticamente significativas $(\mathrm{p}<0,05)$ entre $\mathrm{K}_{a}$ e $\varepsilon_{\mathrm{a}}(\mathrm{r}=0,33)$ e Ds e $\varepsilon_{\mathrm{a}}(\mathrm{r}=-0,68)$ foram observadas. O aumento da Ds normalmente provocado pela compactação causa alterações na estrutura do solo, alterando o tamanho, a distribuição, a continuidade, o volume e a geometria dos poros (Lima et al., 2005) e reduzindo a $\varepsilon_{\mathrm{a}}$ e a $\mathrm{K}_{a}$ em um dado conteúdo de água (Silva et al., 2009). Também, os resultados de Roseberg \& McCoy (1990), Sweeney et al. (2006) e Resurreccion et al. (2007) indicam o quanto $\mathrm{K}_{a}$ é fortemente dependente da condição estrutural do solo. Esses resultados sugerem que o aumento da Ds reduz o espaço poroso disponível para as trocas de gases, o qual é ampliado com o secamento do solo, conforme sugere van Lier (2001).

Índices de continuidade de poros podem ser estabelecidos a partir de regressões lineares entre o $\log \mathrm{K}_{a}$ e $\log \varepsilon_{\mathrm{a}}$ (Ball et al., 1988). As relações entre $\log \mathrm{K}_{a}$ e $\log \varepsilon_{\mathrm{a}}$ para as diferentes camadas e tratamentos estudados são mostradas da figura 4. A inclinação da reta associada a essas duas variáveis indica a habilidade do solo em conduzir gases. No tratamento testemunha e no tratamento de $7 \mathrm{~cm}$ há nítida diferença nas relações entre $\mathrm{K}_{a}$ e $\varepsilon_{\mathrm{a}}$ entre as camadas estudadas, mostrando que na camada de $0-7,5 \mathrm{~cm}$ o sistema poroso estabeleceu maior $\mathrm{K}_{a}$ com o incremento em $\varepsilon_{\mathrm{a}}$ nesses tratamentos. Nos outros tratamentos, verifica-se que há paralelismo entre as camadas, sugerindo que os diferentes volumes de poros estabelecem diferentes valores de $K_{a}$, mas sem distinção quanto à eficiência do sistema poroso. A maior inclinação da reta na camada superficial para o tratamento testemunha deve-se à ausência de pisoteio e consequente manutenção de um sistema poroso contínuo e conectado. Para o tratamento de $7 \mathrm{~cm}$, a inclinação semelhante à da testemunha é reflexo da concentração do sistema radicular agressivo e fasciculado do azevém na superfície do solo e da maior intensidade de secagem e umedecimento, que favoreceu a formação de poros.

Os índices de continuidade de poros (ICP) estabelecidos a partir da figura 4 foram de 2,82 , $2,08,1,52,3,00$ e 2,88 para os tratamentos de 7,14 , 21 e $28 \mathrm{~cm}$ e a testemunha, respectivamente, na camada de $0-7,5 \mathrm{~cm}$. Para a camada de $7,5-15 \mathrm{~cm}$, os valores de ICP foram de 1,45, 1,53, 0,98, 2,50 e 1,12 , respectivamente, para os tratamentos de 7,14 , $21 \mathrm{e} 28 \mathrm{~cm}$ e a testemunha. Os valores de ICP foram maiores na camada de $0-7,5 \mathrm{~cm}$ do que na de $7,5-$ $15 \mathrm{~cm}$, indicando melhor continuidade do sistema poroso. Na camada de $0-7,5 \mathrm{~cm}$, os menores valores de ICP ocorreram nos tratamentos de 14 e $21 \mathrm{~cm}$, comparados aos outros. O tratamento de $7 \mathrm{~cm}$, nesta camada, apresentou valor de ICP muito próximo ao da testemunha, o que pode estar ligado à intensa renovação radicular da maior quantidade de plantas de azevém com sistema radicular fino e agressivo, reduzindo os efeitos deletérios do pisoteio animal no tratamento de $7 \mathrm{~cm}$. Apesar disso, as indicações de campo mostram maior impedimento mecânico

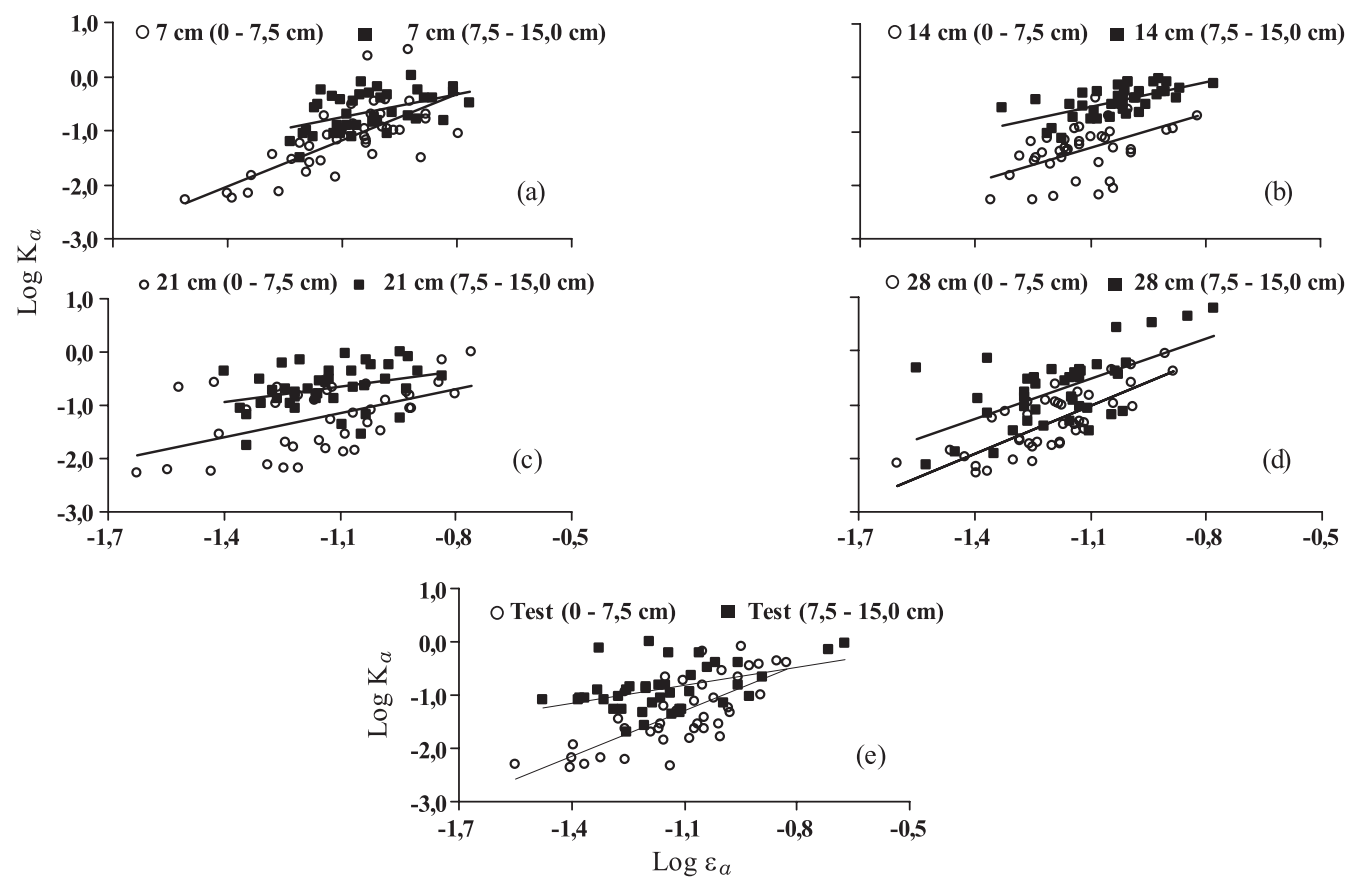

Figura 4. Regressões de $\log K_{a}$ por $\log \varepsilon_{a}$ nas camadas de 0-7,5 e 7,5-15 cm para as alturas de pastejo de 7 (a), 14 (b), 21 (c) e $28 \mathrm{~cm}$ (d) e a testemunha (e). 
neste tratamento na superfície, o que anula a continuidade de poros criada pelas raízes de azevém, as microfendas e as fissuras geradas no solo pelos ciclos de secagem e umedecimento. No tratamento de $28 \mathrm{~cm}$, ao final do ciclo de pastejo verificou-se que a pastagem era composta basicamente por plantas de aveia, porém o amortecimento do pisoteio em função da maior massa vegetal sobre a superfície do solo neste tratamento e a presença de plantas de azevém crescendo com mínimas restrições podem justificar o maior valor de ICP.

Apesar da semeadura simultânea e mesma quantidade de semente nos diferentes níveis, o estabelecimento da aveia ocorre antes e, com isso, há maior supressão do efeito do azevém. Assim, os tratamentos testemunha, 21 e $28 \mathrm{~cm}$ apresentam maior desenvolvimento da aveia do que os de $7 \mathrm{e}$ $14 \mathrm{~cm}$, porém no tratamento de $21 \mathrm{~cm}$ o ICP pode ser menor devido à menor quantidade de forragem para atenuar o efeito do pisoteio, em comparação com a testemunha e o de $28 \mathrm{~cm}$. Esses resultados estão de acordo com os de Silva et al. (2000), os quais indicam que o aumento da massa de aveia e azevém atenua o efeito do pisoteio sobre os atributos físicos do solo.

As cargas impostas ao solo por máquinas agrícolas podem promover compactação em subsuperfície, diminuindo possivelmente o ICP desta. Apesar de o ICP ser menor na segunda camada, a $K_{a}$ é maior. Isso pode ser explicado pelo fato de o índice de continuidade de poros fornecer um valor médio.
Contudo, para um alto valor de $\mathrm{K}_{a}$ é necessário ao menos que um ou poucos poros contínuos atravessem a extensão da amostra. Na segunda camada é mais provável que ocorra essa condição, pois a menor mobilização do solo confere maior estabilidade de agregados e diâmetro médio geométrico (Vieira \& Muzilli, 1984; Ros et al., 1996), acarretando melhor estruturação do solo, o que possibilita a obtenção de altos valores de $\mathrm{K}_{a}$ mesmo com baixo valor de ICP.

Os resultados indicaram que os maiores valores médios de resistência do solo à penetração $(\mathrm{RP})$ foram constatados no tratamento de $7 \mathrm{~cm}$, seguido pelos de 14, 21 e $28 \mathrm{~cm}$ e da testemunha (Figura 5). No entanto, diferenças estatísticas (não sobreposição dos limites superior e inferior dos intervalos de confiança das médias) foram encontradas apenas entre a testemunha e os tratamentos pastejados até a profundidade de $15 \mathrm{~cm}$ e entre o de $7 \mathrm{~cm}$ e os tratamentos de 21 e $28 \mathrm{~cm}$ até $5 \mathrm{~cm}$. Com exceção das camadas de $0-10$ e $10-20 \mathrm{~cm}$ da testemunha, todas as outras apresentaram valores de RP acima de 2,5 $\mathrm{MPa}$. Quanto aos tratamentos pastejados nas profundidades entre 10 e $40 \mathrm{~cm}$, os valores de RP ficaram entre 2,5 e 4,0 $\mathrm{MPa}$, enquanto na testemunha a variação foi de 2,0 a $2,5 \mathrm{MPa}$ para a mesma profundidade.

Foram encontrados valores médios de RP de $4,46,4,09,3,37$ e 2,96 $\mathrm{MPa}$ para as alturas de pastejo de $7,14,21$ e $28 \mathrm{~cm}$, na camada entre 5 e $10 \mathrm{~cm}$ de profundidade. As RPs mais elevadas

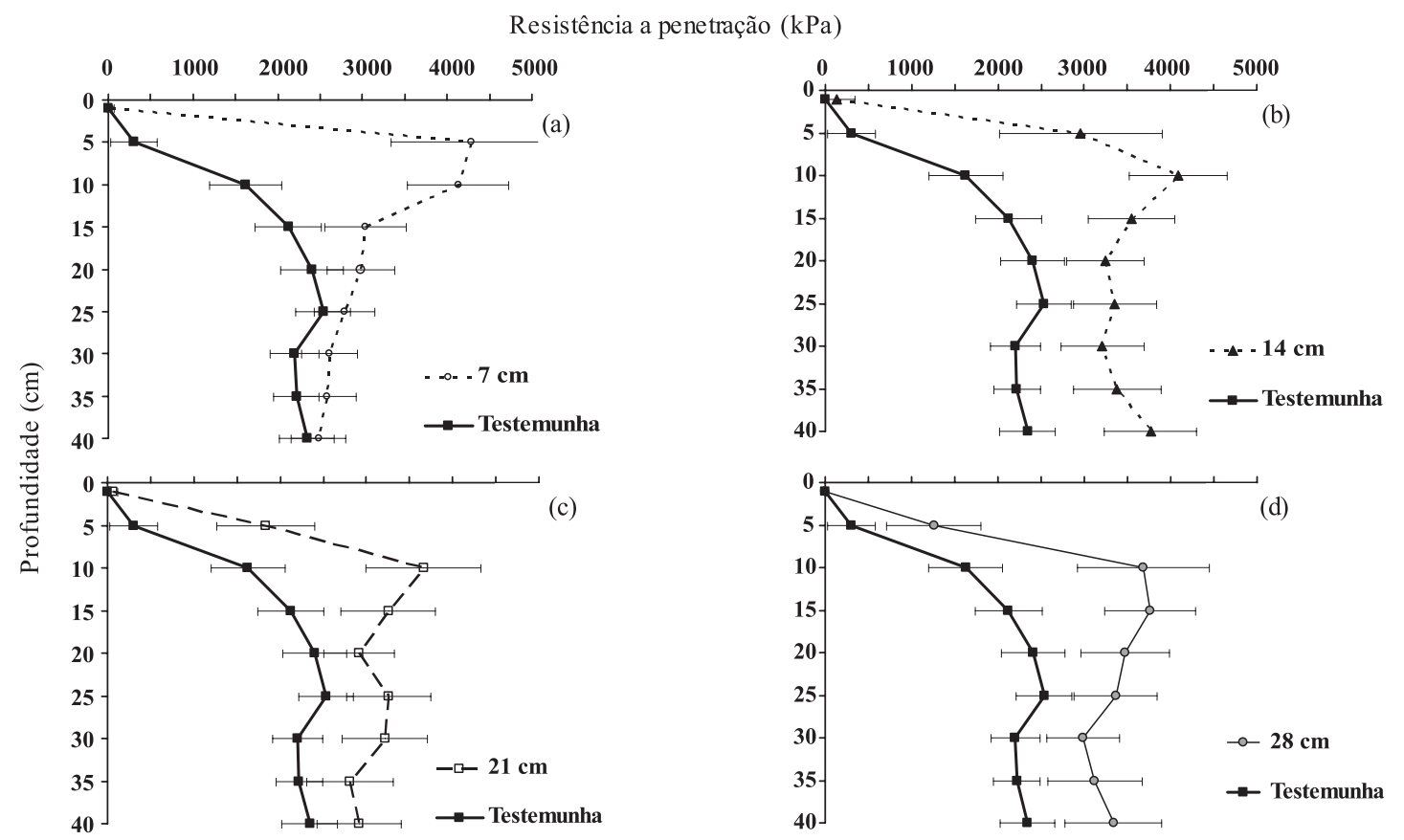

Figura 5. Resistência do solo à penetração (RP) para as alturas de pastejo de 7 (a), 14 (b), 21 (c) e $28 \mathrm{~cm}$ (d), comparadas com a testemunha, até a profundidade de $40 \mathrm{~cm}$. As barras referem-se ao intervalo de confiança da média, e a sobreposição dos intervalos de confiança indica ausência de diferenças entre os tratamentos. 
na camada superficial dos tratamentos pastejados coincidem com valores de conteúdo de água do solo mais baixos para a respectiva camada, em comparação com a testemunha (Quadro 1). Contudo, a ausência de diferenças no conteúdo de água no solo entre os tratamentos pastejados mostra que o aumento da RP principalmente no tratamento de $7 \mathrm{~cm}$, em comparação com os de 21 e $28 \mathrm{~cm}$ (onde houve diferença significativa na camada de $5 \mathrm{~cm}$ ), é resultado da intensificação do pastejo. $\mathrm{O}$ aumento da $\mathrm{RP}$ com a redução da altura de pastejo também foi constatado por Conte et al. (2007) em estudo realizado com SILP num Latossolo Vermelho distroférrico.

A diminuição dos valores médios de Ds nos tratamentos de 7, 14 e $21 \mathrm{~cm}$ após oito anos de SILP pode refletir a capacidade do sistema em promover melhoria na qualidade física do solo. Os resultados da avaliação da Ds não mostraram o resultado esperado, no qual a Ds aumentaria com o aumento da intensidade de pastejo. Em contrapartida, os resultados de RP evidenciaram as alterações estruturais do solo decorrentes das diferentes intensidades de pastejo, notadamente nos diferentes conteúdos de água obtidos nos tratamentos (Quadro 1), pois houve variação apenas na altura e intensidade de pastejo; contudo, o conteúdo de água na testemunha foi superior ao dos tratamentos pastejados, e isso refletiu nos valores de $\mathrm{RP}$ encontrados (Figura 5), resultando em menores valores de RP para a testemunha, em relação a todos os tratamentos pastejados até a profundidade de $15 \mathrm{~cm}$.

De acordo com Klein et al. (2010), a estrutura do solo é um dos principais fatores que exercem influência na retenção de água no solo. Essa afirmação corrobora o resultado obtido, no qual a ausência de pisoteio conferiu à testemunha o maior conteúdo de água, culminando no menor valor de RP. O tratamento de $7 \mathrm{~cm}$, por sua vez, apresentou a maior RP, devido à alteração estrutural causada pelo maior pisoteio animal entre os tratamentos.

\section{CONCLUSÕES}

1. Após oito anos de sistema integração lavourapecuária, a densidade do solo (Ds), a porosidade de aeração $\left(\varepsilon_{\mathrm{a}}\right)$ e a permeabilidade do solo ao ar $\left(\mathrm{K}_{a}\right)$ não confirmaram a hipótese de que a intensificação do pisoteio animal reduz a qualidade física do solo.

2. A resistência do solo à penetração $(\mathrm{RP})$ mostrou que o tratamento de $7 \mathrm{~cm}$ foi estatisticamente mais restritivo que os de 21 e $28 \mathrm{~cm}$ até a profundidade de $5 \mathrm{~cm}$, indicando que a maior intensidade de pastejo resulta em redução da qualidade física do solo em superfície e que a RP apresentou maior sensibilidade na detecção de alterações físicas entre os tratamentos.

\section{LITERATURA CITADA}

AHUJA, L.R.; NANEY, J.W.; GREEN, R.E. \& NIELSEN, D.R. Macroporosity to characterize spatial variability of hydraulic conductivity and effects of land management. Soil Sci. Soc. Am. J., 48:699-702, 1984.

ALBUQUERQUE, J.A.; SANGOI, L. \& ENDER, M. Efeitos da integração lavoura-pecuária nas propriedades físicas do solo e características da cultura do milho. R. Bras. Ci. Solo, 25:717-723, 2001.

BALBINOT JUNIOR, A.A.; MORAES A.; VEIGA, M.; PELISSARI, A. \& DIECKOW, J. Integração lavoura-pecuária: Intensificação de uso de áreas agrícolas. Ci. Rural, 39:19251933, 2009.

BALL, B.C. \& HUNTER, R. The determination of water release characteristics of soil cores at low suctions. Geoderma, 43:195-212, 1988.

BALL, B.C. \& ROBERTSON, E.A.G. Effects of uniaxial compaction on aeration and structure of ploughed or direct drilled soils. Soil Till. Res., 31:135-148, 1994.

BALL, B.C. Modeling of soil pores as tubes using gas permeabilities, gas diffusivities and water release. J. Soil Sci., $32: 465-481,1981$.

Quadro 1. Conteúdo de água ( $\left.\mathrm{kg} \mathrm{kg}^{-1}\right)$ nas distintas camadas do solo sob diferentes alturas de pastejo

\begin{tabular}{|c|c|c|c|c|c|c|c|c|}
\hline \multirow{3}{*}{ Tratamento } & \multicolumn{8}{|c|}{ Profundidade $(\mathrm{cm})$} \\
\hline & \multicolumn{2}{|c|}{$0-10$} & \multicolumn{2}{|c|}{$10-20$} & \multicolumn{2}{|c|}{$20-30$} & \multicolumn{2}{|c|}{$30-40$} \\
\hline & $\mathbf{u}$ & IC & $\mathbf{u}$ & IC & $\mathbf{u}$ & IC & $\mathbf{u}$ & IC \\
\hline 7 & 0,280 & 0,008 & 0,300 & 0,015 & 0,320 & 0,005 & 0,340 & 0,005 \\
\hline 14 & 0,290 & 0,015 & 0,300 & 0,005 & 0,320 & 0,014 & 0,330 & 0,014 \\
\hline 21 & 0,290 & 0,013 & 0,290 & 0,021 & 0,320 & 0,019 & 0,320 & 0,011 \\
\hline 28 & 0,290 & 0,006 & 0,280 & 0,023 & 0,320 & 0,009 & 0,330 & 0,012 \\
\hline Testemunha & 0,320 & 0,009 & 0,330 & 0,018 & 0,330 & 0,013 & 0,340 & 0,003 \\
\hline
\end{tabular}

u: conteúdo de água do solo $\left(\mathrm{kg} \mathrm{kg}^{-1}\right)$; IC: intervalo de confiança. 
BALL, B.C.; O'SULLIVAN, M.F. \& HUNTER, R. Gas diffusion, fluid flow and derived pore continuity indices in relation to vehicle traffic and tillage. J. Soil Sci., 39:327-339, 1988.

BERTOL, I.; ALMEIDA, J.A.; ALMEIDA, E.X. \& KURTZ, C. Propriedades físicas do solo relacionadas a diferentes níveis de oferta de forragem de capim-elefante-anão cv. Mott. Pesq. Agropec. Bras., 35:1047-1054, 2000.

BEUTLER, A.N.; SILVA, N.L.N.; CURI, N.; FERREIRA, M.M.; CRUZ, J.N. \& PEREIRA FILHO, I.A. Resistência a penetração e permeabilidade de Latossolo Vermelho Distrófico típico sob sistemas de manejo na região dos cerrados. R. Bras. Ci. Solo, 25:167-177, 2001.

BLACKWELL, P.S.; RINGROSE-VOASE, A.J.; JAYAWAR DANE, N.S.; OLSSON, K.A.; MCKENZIE, D.C. \& MASON, W.K. The use of air filled porosity and intrinsic permeability to characterize structure of macropore space and saturated hydraulic conductivity of clay soils. J. Soil Sci., 41:215-228, 1990.

CLARK, J.T.; RUSSELL, J.R.; KARLEN, D.L.; SINGLETON, P.L.; BUSBY W.D. \& PETERSON, B.C. Soil surface property and soybean yield response to corn stover grazing. Agron. J., 96:1364-1371, 2004.

COLLARES, G.L.; REINERT. D.J.; REICHERT, J.M. \& KAISER, D.K. Qualidade física do solo na produtividade da cultura do feijoeiro num Argissolo. Pesq. Agropec. Bras., 41:1663$1674,2006$.

CONTE, O.; LEVIEN, R.; TREIN, C.R.; CEPIK, C.T.C. \& DEBIASE, H. Demanda de tração em haste sulcadora na integração lavoura-pecuária com diferentes pressões de pastejo e sua relação com o estado de compactação do solo. Eng. Agríc., 27:220-228, 2007.

DÖRNER, J. \& HORN, R. Anisotropy of pore functions in structured Stagnic Luvisols in the Weichselien moraine region in N Germany. J. Plant. Nutr. Soil Sci., 169:213-220, 2006.

EMPRESA BRASILEIRA DE PESQUISA AGROPECUÁRIA - EMBRAPA. Centro Nacional de Pesquisa de Solos. Sistema brasileiro de classificação de solos. Brasília, Embrapa Produção de Informação, 2006. 306p.

FIDALSKI, J.; TORMENA, C.A.; CECATO, U.; BARBERO, L.M.; LUGÃ̃O, S.M.B. \& COSTA, M.A.T. Qualidade física do solo em pastagem adubada e sob pastejo contínuo. Pesq. Agropec. Bras., 43:1583-1590, 2008.

FLORES, J.P.C.; ANGHINONI, I.; CASSOL, L.C.; CARVALHO, P.C.F.; LEITE, J.G.D.B. \& FRAGA, T.I. Atributos físicos do solo e rendimento de soja em sistema plantio direto em integração lavoura-pecuária com diferentes pressões de pastejo. R. Bras. Ci. Solo, 31:771-780, 2007.

GIAROLA, N.F.B.; TORMENA, C.A. \& DUTRA, A.C. Degradação física de um Latossolo Vermelho utilizado para produção intensiva de forragem. R. Bras. Ci. Solo, 31:863-873, 2007.

GRABLE, A.R. \& SIEMER, E.G. Effects of bulk density, aggregate size, and soil water suction on oxygen diffusion, redox potential and elongation of corn roots. Soil Sci. Soc. Am. J., 32:180-186, 1968.
GREGORY, A.S.; WATTS, C.W.; WHALLEY, W.R.; KUAN, H.L.; GRIFFITHS, B.S.; HALLETT, P.D. \& WHITMORE, A.P. Physical resilience of soil to field compaction and the interactions with plant growth and microbial community structure. Eur. J. Soil Sci., 58:1221-1232, 2007.

GROENEVELT, P.H.; KAY, B.D. \& GRANT, C.D. Physical assessment of soil with respect to rooting potential. Geoderma, 34:101-114, 1984.

GROSSMAN, R.B. \& REINSCH, T.G. Bulk density and linear extensibility. In: DANE, J.H. \& TOPP, C., eds. Methods of soil analysis: Physical methods. Madison, Soil Science of Society of America, 2002. p.201-228.

IMHOFF, S.; SILVA, A.P.; DIAS JÚNIOR, M.S. \& TORMENA, C.A. Quantificação das pressões críticas para o crescimento das plantas. R. Bras. Ci. Solo, 25:11-18, 2001.

JONG VAN LIER, Q. Oxigenação do sistema radicular: Uma abordagem física. R. Bras. Ci. Solo, 25:233-238, 2001.

KLEIN, V.A.; BASEGGIO, M.; MADALOSSO, T. \& MARCOLIN, C.D. Textura do solo e a estimativa do teor de água no ponto de murcha permanente com psicrômetro. Ci. Rural, 40:1550-1556, 2010

LANZANOVA, M.E.; NICOLOSO, R.S.; LOVATO, T.; ELTZ, F.L.F.; AMADO, T.J.C. \& REINERT, D.J. Atributos físicos do solo em sistema de integração lavoura-pecuária sob plantio direto. R. Bras. Ci. Solo, 31:1131-1140, 2007.

LAPEN, D.R.; TOPP, G.C.; GREGORICH, E.G. \& CURNOE, W.E. Least limiting water range indicators of soil quality and corn production, Eastern Ontario, Canada. Soil Till. Res., 78:151-170, 2004.

LIMA, H.V.; LIMA, C.L.R.; LEÃO, T.P.; COOPER, M.; SILVA, A.P. \& ROMERO, R.E. Tráfego de máquinas agrícolas e alterações de bioporos em área sob pomar de laranja. R. Bras. Ci. Solo, 29:677-684, 2005.

McCARTHY, K.P. \& BROWN, K.W. Soil gas permeability as influenced by soil gas-filled porosity. Soil Sci. Soc. Am. J., 56:997-1003, 1992.

McQUEEN, D.J. \& SHEPHERD, T.G. Physical changes and compaction sensitivity of a fien-textured, poorly drained soil (Typic Endoaquept) under varying durations of cropping, Manawatu Region, New Zealand. Soil Till. Res., 25:217-230, 2002.

MOOT, G.O. \& LUCAS, H.L. The design conducted and interpretation of grazing trials on cultivated and improved pasture. In: INTERNATIONAL GRASSLAND CONGRESS, 6., 1952, Pensilvânia. Proceedings... Pensilvânia, State College Press, 1952. p.1380-1395.

PAYTON, M.E.; MILLER, A.E. \& RAUN, W.R. Testing Statistical Hypotesis using standard error bars and confidence intervals. Comm. Soil Sci. Plant. Anal., 31:547-551, 2000.

PETEAN, L.P.; TORMENA, C.A. \& ALVES, S.J. Intervalo hídrico ótimo de um Latossolo Vermelho distroférrico sob plantio direto em sistema de integração lavoura-pecuária. R. Bras. Ci. Solo, 34:1515-1526, 2010. 
PETEAN, L.P.; TORMENA, C.A.; FIDALSKI, J. \& ALVES, S.J. Altura de pastejo de aveia e azevém e qualidade física de um Latossolo Vermelho distroférrico sob integração lavoura-pecuária. Semina: Ci. Agric., 30:1009-1016, 2009.

PHILLIPS, R.E. \& KIRKHAM, D. Soil compaction in the Field and corn growth. Agron. J., 54:29-34, 1962.

RESSURECCION, A.C.; KAWAMOTO, K.; KOMATSU, T.; MOLDRUP. P.; OZAKI, N. \& ROLSTON, D.E. Gas transport parameters along field transects of a volcanic soils. Soil Sci., 172:3-16, 2007.

RODRIGUES, S.; SILVA, A.P.S.; GIAROLA, N.F.B. \& ROSA, J.A. Permeabilidade ao ar em Latossolo Vermelho sob diferentes sistemas de manejo. R. Bras. Ci. Solo, 35:105-114, 2011.

ROS, C.O.; LOPES, E.L.L.; SECCO, D. \& PASA, L. Influência do tempo de cultivo no sistema de plantio direto nas características físicas de um Latossolo Vermelho-Escuro. Ci. Rural, 26:397-400, 1996.

ROSEBERG, R.J. \& McCOY, E.L. Measurement of soil macropore air permeability. Soil Sci. Soc. Am. J., 54:969-974, 1990.

ROSEBERG, R.J. \& McCOY, E.L. Tillage and traffic-induced changes in macroporosity and macropore continuity: Air permeability assessment. Soil Sci. Soc. Am. J., 56:12611267, 1992.

SAS INSTITUTE. SAS: User's guide: Statistics. 9.ed. Cary, 2002. $943 p$

SILVA, A.P.; IMHOFF, S. \& CORSI, M. Evaluation of soil compaction in an irrigated short-duration grazing system. Soil Till. Res., 70:83-90, 2003.

SILVA, A.P.; KAY, B.D. \& PERFECT, E. Characterization of the least limiting water range. Soil Sci. Soc. Am. J., 58:17751781, 1994.

SILVA, A.P.; LEÃO, T.P.; TORMENA, C.A. \& GONÇALVES, A.C.A. Determinação da permeabilidade ao ar em amostras indeformadas de solo pelo método da pressão decrescente. R. Bras. Ci. Solo, 33:1535-1545, 2009.

SILVA, V.R.; REINERT, D.J. \& REICHERT, J.M. Susceptibilidade a compactação de um Latossolo Vermelho escuro e de um Podzólico Vermelho-Amarelo. R. Bras. Ci. Solo, 4:239-249, 2000.

SWEENEY, D.W.; KIRKHAM, M.B. \& SISSON, J.B. Crop and soil response to wheel-track compaction of a claypan soil. Agron. J., 98:637-643, 2006.

TARDIEU, F. Analysis of the spatial variability of maize root density. Plant Soil, 107:259-266, 1988.
TAYLOR, H.M.; ROBERTSON, G.M. \& PARKER, J.J. Soil strength root penetration for medium to coarse textured soil materials. Soil Sci. Soc. Am. J., 102:18-22, 1966.

TOPP, G.C. \& FERRE, P.A. Methods for measurement of soil water content: Thermogravimteric using convective oven drying. In: DANE, J.H. \& TOPP, G.C., eds., Methods of soil analysis: Physical methods. Madison, Soil Science Society of America/American Society of Agronomy, 2002. Part 4. p. $422-424$.

TORMENA, C.A.; ARAÚJO, M.A.; FIDALSKI, J. \& COSTA, J.M. Variação temporal do intervalo hídrico ótimo de um Latossolo Vermelho distroférrico sob sistemas de plantio direto. R. Bras. Ci. Solo, 31:211-219, 2007.

TORMENA, C.A.; BARBOSA, M.C.; COSTA, A.C.S. \& GONÇALVES, A.C.A. Densidade, porosidade e resistência à penetração em Latossolo vermelho distrófico sob diferentes sistemas de preparo do solo. Sci. Agric., 59:795-801, 2002.

TORMENA, C.A.; SILVA, A.P. \& LIBARDI, P.L. Caracterização do intervalo hídrico ótimo de um Latossolo Roxo sob plantio direto. R. Bras. Ci. Solo, 22:573-581, 1998.

TORMENA, C.A.; SILVA, A.P.; GONÇALVES, A.C.A. \& FOLEGATTI, M.V. Intervalo ótimo potencial de água no solo: Um conceito para avaliação da qualidade física do solo e manejo da água na agricultura irrigada. Eng. Agric., 3:86-292, 1999.

TREIN, C.R.; COGO, N.P. \& LEVIEN, R. Métodos de preparo do solo na cultura do milho e ressemeadura do trevo, na rotação aveia+trevo/milho, após pastejo intensivo. R. Bras. Ci. Solo, 15:105-111, 1991.

VEIGA, M.; HORN, R.; REINERT, D.J. \& REICHERT, J.M. Soil compressibility and penetrability of an Oxisol from southern Brazil, as affected by long-term tillage systems. Soil Till. Res., 92:104-113, 2007.

VIEIRA, M.J. \& MUZILLI, O. Características físicas de um Latossolo Vermelho-Escuro sob diferentes sistemas de manejo. Pesq. Agropec. Bras., 19:873-882, 1984.

VOGELER, I.; CHICHOTA, R.; SIVAKUMARAN, S.; DEURER, M. \& McIVOR, I. Soil assessment of apple orchards under conventional and organic management. Austr. J. Soil. Res., 44:745-752, 2006.

XU, X.; NIEBER, J.L. \& GUPTA, S.C. Compaction effect on the gas diffusion coefficient in soils. Soil Sci. Soc. Am. J., $56: 1743-1750,1992$. 